Mariusz Affek

\title{
LA SPECIFICITÀ DEL DIRITTO POLACCO GENEALOGICO ED ARALDICO
}

\section{La rivoluzione dell'informazione}

Praticamente ogni paese europeo ha una propria identità culturale formata dalla storia ed lascia l'impronta sui segni e simboli. Lo si collega ad un senso di orgoglio nazionale ed anche familiare. A questo fabbisogno universale rispondono scienze ausiliarie della storia-in particolare genealogia ed araldica. Entrambi questi settori del sapere non solo sono molto spettacolari" per la possibilità di creare alberi genealogici e stemmi, ma anche si integrano perfettamente con il movimento mondiale genealogico-araldico che è apparso alla fine degli anni ' 80 e ' 90 del XX secolo insieme con l'invenzione del computer personale e dell' internet. Questa rivoluzione dell'informazione ha dato l'occasione per creare interamente nuove modalità di raccolta, archiviazione e presentazione dei fatti sul tema della provenienza dei clan, delle famiglie o delle loro arme'. Parallelamente a questo fenomeno c'era "l'onda" di nuove pubblicazioni genealogiche ed araldiche (colorate e popolari) che hanno ancora molti acquirenti interessati per testare le proprie "radici" familiari"

Ora genealogisti polacchi utilizzano un gran numero di programmi informatici professionali per la conservazione di raccolta e per lo sviluppo di dati genealogici come: "Geno Pro", "FZip Family Tree", "Genius Family Tree", "History Family Genbox", "Gen Designer", "Brother's Keeper", "Familia Builder", "PAF" ecc. Purtroppo, la maggior parte di loro sono versioni polacche di programmi gia esistenti all'Occidente. II più delle volte usata è il programma polacco "L'Albero Genealogico" - continuamente in evoluzione.

Una vera "eruzione" di tali pubblicazioni era negli anni ' 80 e ' 90 del secolo $X X$, quando gia si può ufficialmente risalire ai propri antenati e quando quest' occupazione non si tratta come un elemento della lotta ideologica contro il regime attuale. In quello periodo si formavano molte società genealogiche-araldiche, ma attualmente la maggioranza di loro è già eliminata a causa della mancanza di persone e di risorse. In quegli anni sono state emesse tali pubblicazioni quali: 1) B. Czarnecki, "Heraldyka", T. I-III, Varsavia 1983-1984; 2) J. Łojko, "Średniowieczne herby polskie”, Posnania 1985; 3) A. Kulikowski, „Heraldyka szlachecka”, Varsavia 1990; 4) J. Piechowski, "Ukryte światła herbów", Varsavia 1991; 5) R. T. Prinke, "Poradnik genealoga amatora", Varsavia 1992; 6) J. Szymański, "Herbarz średniowiecznego rycerstwa polskiego", Varsavia 1993; 7) S.K. Kuczyński, "Polskie herby ziemskie. Geneza, treści, funkcje", Varsavia 1993; 8) J. Piechowski, "Herby, magia, mity", Varsavia 1995; 9) W.J. Walat, "Rodzinne korzenie czyli wywód genealogiczny", Resovia 1996; 10) T. Zielińska, "Poczet polskich rodów arystokratycznych", Varsavia 1997 (con le tavole genealogiche); 11) J. Kołyszko, "I ty potrafisz narysować drzewo genealogiczne", Danzica 1997 (libro per i bambini); 12) P. Dudziński, "Alfabet heraldyczny", Varsavia 1999. Tuttavia anche nel XXI secolo dura una "moda" per godere i nostri occhi delle opere con una splendida grafica degli stemmi. Per esempio: 1) T. Gajl, "Herby szlacheckie Rzeczypospolitej Obojga Narodów”, Danzica 


\section{La genesi dello stemma in Polonia}

Occorre ricordare che queste scienze ausiliarie della storia abbiano molti elementi comuni con la legge, perché non si può creare gli stemmi e studiare una storia dei suoi antenati senza il sapere delle regole stabilite molti secoli fa. Inoltre in Polonia dei primi secoli del Medioevo c'erano stati alcuni importanti processi sociali che in Europa Occidentale hanno proceduto in modo diverso e più rapidamente. In conseguenza tale fenomeno ha portato alla creazione di caratteristiche specifiche di genealogia e d'araldica polacca. Questo si riferisce ad un senso di legami con la comunità della stirpe (che possedeva un comune antenato) e significava che i primi cavalieri polacchi sentivano più fortemente associati con la loro stirpe che con la loro terra. In risultato i nostri cavalieri prendevano i terreni ed i privilegi individuali più raramente che i loro "colleghi" dell'Europa Occidentale. Tale situazione ha portato ad un diritto feudale meno complicato ed a minore numero di vassalli e-nel successivo periodo- alla chiusura dello stato nobile sulla base di stirpe-non di terra. Questo ha avuto le conseguenze vaste per l'araldica polacca, perché mentre all'Occidente lo stemma era usato per tutta la famiglia, in Polonia la stessa funzione era legata soprattutto alla stirpe ${ }^{3}$.

Quindi nel Medioevo sulle terre polacche erano noti solo circa 200 immagini di stemmi, invece nello stesso tempo nel territorio della lingua tedesca hanno funzionato più di 200 mila tali simulacri d'armi. Inoltre in Polonia é stata creata una distribuzione atipica dei ceppi sui familiari (seduti) e sugli araldici (collegati per uno stemma). Nel primo caso era visibile cosi la comunità di sangue come la comunità di blasone, ma nel secondo caso era già esclusivamente la stessa immagine di stemma-senza nessuna unione di sangue che all'Occidente si sembra essere un fenomeno sconosciuto. Allora in qual modo hanno preso origine le stirpi d'armi in Polonia? Molto spesso durante il servizio militare sulla casa signorile i cavalieri minori hanno accettato il stemma del suo padrone, rinunziando al proprio blasone. Un altro fattore potrebbe essere una comune origine etnica-come nel caso del blasone "Sas" che raccoglieva i cavalieri d'origine sassone venuti a noi dalla zona di Slovacchia e di Transilvania (illustr. nr 1). Allo stesso modo é stato creato uno stemma "Prussia" usato per i cavalieri polacchi fuggiti dal territorio dello Stato dei Cavalieri Teutonici. In Polonia c'erano perfino tali situazioni quando qualche centinaio delle famiglie hanno usato lo stesso stemma "Jelita" o "Nałęcz", peró queste famiglie non possedevano nessuni legami di sangue fra loro, ma facevano riferimento al comune ante-

2003; 2) R.T. Komorowski, "llustrowany przewodnik heraldyczny", Pelplin-Varsavia 2007 o 3) A. Znamierowski, „Wielka księga heraldyki”, Varsavia 2008. Nell'orientamento dell'offerta editoriale aiuta il libro di A. Celej, "Bibliografia genealogii i heraldyki polskiej za lata 1980-2001", Varsavia 2006. ska w okresie rozdrobnienia feudalnego", ed. H. Łowmiański, Breslavia-Varsavia-Cracovia-Danzica 1973, pp. 162-168; A. Wyrobisz, „Polska heraldyka nowożytna. Na pograniczu historii społecznej, historii sztuki i historii literatury”, [in:] „Heraldyka i okolice”, ed. A. Rachuba, S. Górzyński i H. Manikowska, Varsavia 2002, p. 133. 
nato leggendario. Nello stesso periodo in Italia solo una famiglia unita per i legami documentati e reali di sangue poteva usare il blasone degli Orsini o degli Colonna ${ }^{4}$.

Molti studiosi dicono che l'elmo è stato fatto in relazione a cambiamenti nell' armatura del cavaliere ed a comparsa d'elmo nella forma cilindrica (pentolare). Tale casco rendeva impossibile per vedere la faccia del cavaliere, perció c'è stato necessario porre segni distintivi sullo scudo. Questa pratica divenne popolare nel corso del XII secolo ed appunto di questo periodo provengono più vecchie stemmi conosciute e documentate in Occidente-soprattutto da Francia e da Germania. Tuttavia mentre nella maggior parte delle lingue dell'Europa Occidentale la parola "stemma" si unisce chiaramente con la parola "arma" (in francese-“armoiries", in inglese-“arms", in tedesco-"Wappen" (dalla definizione"Waffen"), nel caso della lingua polacca e ceca "stemma" (in ceco-"erb") deriva dalla parola tedesca "Erbe" che significa "eredità". Così in entrambe queste associazioni riflettono due caratteri distintivi dello stemma: la sua genesi militare e la sua ereditarietà almeno nelle tre generazioni. Inoltre c'erano ancora altre condizioni necessarie per il segno concreto trattare come stemma, cioè: emblema visto sullo scudo (non sul sigillo e non sulla bandiera) e la presenza degli stessi emblemi geometriche e figurative con lo stesso stile. Tuttavia tali requisiti non hanno già trovato il suo riflesso nella morfologia ${ }^{5}$.

In Polonia emblemi araldici si formavano più tardi che in Occidente- fra la metà del XIII secolo (Slesia) e la metà del XIV secolo (Mazovia). Primi esempi dell'uso degli stemmi concreti gentilizi sono: "Topór" (la fine del XIII secolo), "Lis" e "Rawa" (entrambi nel 1306) ${ }^{6}$. La base per la formazione degli stemmi ereditari erano così vecchi segni militari (tipo d'europeo-occidentale) come i marchi d'identificazione nella forma dei righi (tipo locale). Questi ultimi hanno soprattutto l'origine slava e c'erano scolpiti sui tronchi degli alberi, sui vasi di ceramica, sulle urne e sulle armi. Hanno avuto un carattere della runa e perfino di segni visti sul totem magico. Anche oggi non possiamo identificare alcuni di loro (illustr. nr 2). Invece altri marchi erano trasformati gradualmente in oggetti compatibili con araldica europea e sono conosciuti come-per esempio- "le travi" (stemma "Abdank" o "Dębno"), "i fiumi" (stemma "Szreniawa"), "le spade" (stemma "Herburt"), "le bandiere" (stemma "Radwan") o "le frecce stilizzate" (stemma "Sas")

\footnotetext{
Vedi: J. Bieniak, op. cit., pp. 162-210, ma anche W. Dworzaczek, "Genealogia”, Varsavia 1959, pp. 15-18. A. Znamierowski, op. cit., pp. 21-23.

J. Bieniak, „Heraldyka polska przed Długoszem”, [in:] „Sztuka i ideologia XV w.”, ed. P. Skubiszewski, Varsavia 1978, p. 187.

7 W. Semkowicz, „Encyklopedia nauk pomocniczych historii”, Cracovia 1945, pp. 171-175; ibidem, "Godła napieczętne a herby szlachty polskiej wieków średnich”, [in:] „Sprawozdania z czynności i posiedzeń Akademii Umiejętności”, T. XIX (1914), nr 2, pp. 10-12; S.K. Kuczyński, „Niektóre zagadnienia symboliki heraldycznej na tle funkcjonowania herbu jako znaku”, [in:] „Problemy nauk pomocniczych historii”, ed. J. Szymański, T. II, Katowice 1973, pp. 34-35; J. Bieniak, „Heraldyka polska...,, op. cit., p. 187; A. Kulikowski, op. cit., pp. 19-20.
} 


\section{La divisione d'emblemi araldici polacchi}

Su questa base J. Szymański ha proposto la divisione d'emblemi araldici polacchi in tre gruppi: 1)quelli che derivano direttamente dall' araldica occidentale, 2) sono adatti solo all' araldica polacca, 3) con elementi caratteristici per l'Europa Occidentale e tipicamente polacchi. Il primo gruppo è composto da solo le armi che sono qualificati come figure geometriche (denominate "onorevoli") ed anche da quelli dove esiste una ripartizione del campo dello scudo ${ }^{8}$.

Ad Ovest già soli campi vuoti (bianchi) erano stati in grado di creare il loro emblema araldico, mentre in Polonia erano per lo più pieni d'emblemi. Nel nostro paese conosciamo solo 10 figure geometriche occidentali, ma anche ulizzati per pubblicare qualche elemento aggiuntivo su di loro. Tra queste figure occupano un posto preminente diverse varianti della croce, peró prevalentemente loro non sono connessi con il simbolismo religioso. La maggior parte di queste croci è stata creata da vecchi marchi locali di proprietà. C'erano anche quelle situazioni in cui la croce nello stemma si trasforma in una spada come risultato della lunga evoluzione dell'iconografia.

Nel caso del mondo animale presente agli stemmi della cavalleria polacca era il fatto caratteristico che non si usava l'aquila coronata mai a causa della sua somiglianza a quella delle armi di Piast (della dinastia regnante in Polonia) e poi del Regno Polacco. Eppure i cavalieri hanno dovuto presentare un rispetto per la dinastia a cui erano collegati per le relazioni feudali. Invece il mondo dei rettili rappresentava solo unico serpente (perfino coronato ed attribuito alla famiglia italiana Sforza) del blasone "Wężyk". Nel gruppo degli animali chimerici negli stemmi polacchi sono apparsi solo un grifone ed un unicorno e tre appropriate solo per la nostra idea araldica: mezzo grifone con la coda di storione (stemma "Pobędzie"), mezzo cinghiale e mezz'orso (stemma "Sokola") e l'aquila con la testa d'un gallo (stemma "Trach"). Infine il tema dell'uomo e delle sue parti del corpo sono stati utilizzati-proprio come in Occidente-piuttosto raramente, anche se ci sono state le immagini in modo originale come Mauro e Negra-non senza l'influenza d'araldica francese e tedesca.

Non meno interessante si sembra la situazione con il regno vegetale scarsamente rappresentato nell' araldica polacca. Qui possiamo vedere solo due fiori: rosa e giglio e tre alberi: quercia, pino e melo. Nel caso del giglio abbiamo perfino il suo tipo angioino (d'Angiò) in quattro stemmi. Lo non stupisce per riguardo al regno di questa dinastia francese in Polonia negli anni 1370-1399 (il re d'Ungheria Luigi d' Angiò e la sua figlia Santa Edvige Regina). 
Le idee dell'universo hanno riflesso soprattutto i corpi celesti rappresentati nei blasoni. Qui ha dominato il sole, ma la luna non è stato presentato mai in plenilunio (sempre in una fase simile a novilunio ed in una posizione innaturale). C'era anche la stella esagonale (ettagonale -solo nel stemma "Stembark").

Nel gruppo polacco di figure artificiali (cioè non derivati dal mondo naturale, ma prodotti per le mani degli uomini) hanno dominato tutti gli oggetti associati ad un tipico sequestro dei cavalieri - cioè alla condotta della guerra ed alla cattura degli animali, ma anche ai lavori in corso per la fattoria (ad esempio: rastrello, forcone ed altri attrezzi). Si riconosce che la maggior parte di questi soggetti (perfino l'arma) aveva la provenienza polacca, peró sotto l'influenza dei modelli occidentali. Anche caratteristica é una povertà delle costruzioni militari nell'araldica polacca: loro sono effettivamente limitati alla torre ed alle mura- del resto non particolarmente distinte per la sua architettura.

Finalmente occorre elencare stemmi polacchi con gotiche lettere latine sullo scudo. La loro origine sembra essere appunto d'Ovest e non polacca9.

\section{Le regole araldiche per blasonare (descrivere) le armi}

Tuttavia il più interessante dal punto giuridico di vista sono le regole araldiche che descrivano come occorre blasonare cioè descrivere le armi. Ad Ovest si è fatto spesso ed è ancora soprattutto in francese, invece l'araldica polacca ha affermato questi principi solo nel XX secolo sul suo terreno- tutto allo scopo di ottenere la descrizione più accurata, ma una breve e senza parole inutili. Quindi:

1) Durante la descrizione del blasone sempre si ricorda che i termini "lato destro" e "lato sinistro" si utilizza dalla posizione del cavaliere che sta in piedi da dietro lo scudo -cioè diversamente che nel caso di una persona che guarda il disegno di tale stemma.

2) La descrizione del blasone sempre si comincia dal campo dello scudo e poi si passa a descrivere l'emblema e la gemma. Se lo scudo è diviso in due campi in palo (verticalmente), la descrizione si inizia dal campo destro (araldicamente). Nella situazione, quando lo scudo è diviso in due campi in cintura (orizzontale), cominciamo a blasonare il campo superiore. 
3) Si suppone che di solito l'emblema abbia una posizione centrale sullo scudo. Pertanto se questa posizione non è in qualunque modo modificata, non si pubblica tali dati nella descrizione.

4) Nel caso dei sistemi simmetrici l'emblema è rivolto sempre a destro (araldicamente) lato dello scudo. Quindi nella descrizione si considera la direzione soltanto allorchè le figure sono rivolte inaraldicamente-cioè a sinistra. In quel tempo abbiamo a fare con cosiddetto "lo sforzo di blasone"- cioè con una ricevuta della forma meno onorevole. Anche si deve descrivere tale situazione quando lo scudo è rivolto in un gesto di cortesia verso un altro stemma vicino o verso un gruppo degli stemmi. Questo è "il saluto araldico".

5) La ripetizione molteplice della stessa figura nel campo dello scudo (per esempio nello stemma "Doliwa" dove ci sono tre rose) non genera "lo sforzo di blasone".

6) Un gruppo d'oggetti inanimati si sempre caratterizza la posizione statica (per esempio: si riporre la luna con gli spicchi in su e la freccia con punta acuta anche in su), dunque non si indica tali dettagli nella descrizione- a meno che non vi sia un cambiamento di tale posizione naturale.

7) Alcuni animali, piante ed edifici hanno già fisso aspetto esterno e perció si dice di loro che siano "araldicamente stilizzati". In tal caso non bisogna descriverli.

Tale descrizione- come vediamo- tiene conto soprattutto l'immagine dello scudo e dell'emblema, invece elude l'elmo e la corona (se questa non c'è di barone, di conte o una mitra ducale). Inoltre si generalizza (o di tanto in tanto anche si elude) altri elementi del blasone come: gemme, lambrecchini ( 0 svolazzi) ed eventualmente medaglie, motto d'araldica e figure sostenenti lo scudo ${ }^{10}$.

In questo modo veniamo agli altri elementi del blasone che in araldica polacca possedono certe note caratteristiche. E' noto bene un fatto che l'elmo araldico creava come l'immagine del copricapo di cavaliere, ma in Polonia c'era la sua forma di base conosciuta come il casco nella forma cilindrica (pentolare). Tale modello velava completamente la testa del cavaliere ed aveva solo strette fessure per gli occhi e fori da respirare. Esso è diventato popolare alla metà del XIV secolo e da quello tempo cominciò ad essere utilizzato anche nei nostri stemmi. La sua versione chiamata "bocca di rana" (usata nei tornei ed offerta un più grande campo di vista) deriva già dall'inizio del XV secolo, ma simultaneamente erano usati anche gli elmi di barracon un caratteristico traliccio (vedi: confronto di questi tre tipi degli elmi- illustr. nr 3). Purtroppo, non si è formata nessuna regola polacca per usare gli elmi nei blaso- 
ni ed in conseguenza abbiamo i nostri stemmari pieni d'immagini del solo casco di barra. Non si prende in considerazione nessune date dell'origine di questi-copricapi in prospettiva della loro evoluzione storica. A vuoto si puó cercare una diversità di posizione dell'elmo ("en face" o di profilo), suo colore, un' identificazione del tipo di metallo e perfino un numero di barre nell'elmo con il nome adeguato. Inoltre non sempre tale casco ha avuto adeguate proporzioni in confronto alle dimensioni dello scudo ${ }^{11}$.

Il secondo più importante elemento (dopo l'emblema) dello stemma polacco erano le gemme (conosciute anche come "cimieri" dal francese). Di solito erano trattate esclusivamente come ornamento dell'elmo e qualche volta questo termine era usato erroneamente per definire tutto casco. In ogni caso nella nostra araldica c'era la libertà assoluta nella presentazione delle gemme e sono stati solo tentativi di loro classificazione ${ }^{12}$.

Si puó distinguere quattro tipi delle gemme:

1) tautologiche o identiche con l'emblema sullo scudo-per esempio le stemme: "Dąb", "Kolumna", "Labędź”, "Starykoń”, "Topór", "Rogala” ecc.;

2) tautologiche, ma con decorazioni aggiunti che spesso sono ridotti ai flabelli od alle piume singole di pavone, di struzzo o di gallo, inoltre alle ali imprecisate, alle corna di cervo e perfino alle stelle di sprone cavalieresco;

3) contenenti tali elementi decorativi come le piume ed i flabelli (e questo è il più grande dei nostri gruppi delle gemme);

4) associate ad un nome o ad un proclama dello stemma: in tali casi il nome o il proclama richiama alla mente un concreto oggetto araldico che simultaneamente è presentato come l'immagine nella gemma- per esempio le stemme "Jastrzębiec" o "Lis".

Oltre alla gemma fanno parte degli ornamenti dell'elmo anche tal elementi quali: corona araldica (incoronata del casco) e lambrecchini 0 svolazzi (nastri bicolori caduti dalla corona). L'araldica dell' Europa Occidentale conosceva altri elementi come: tenenti (cioè figure che "sostengono" lo scudo), manto (impropriamente anche padiglione) che circonda uno stemma e divisa (di solito latina) che esprime una massima collocata sui nastri sotto lo scudo. Tuttavia nel caso dell'araldica polacca questi elementi non hanno trovato l'approvazione generale- ad eccezione di poche famiglie titolate e straniere residenti in Polonia. Inoltre nel nostro paese solo nel XVIII secolo (non senza l'influenza dell'ideologia illuminista) si diffuse l'abitudine

11 A. Kulikowski, op. cit., pp. 176-181.

12 Ibidem, p. 188; A. Heymowski, „Les cimiers médiévaux polonaise”, [in:] "Recueil du IX Congrès international des sciences genealogique et héraldique”, Berne 1968, pp. 131 e seg.; J. Szymański, op. cit., pp. 50-60. 
d'aggiungere ai propri stemmi i segni delle funzioni ed uffici laici esercitati in momento concreto (in araldica ecclesiastica c'era il costume già dal XV secolo). Così agli stemmi sono stati aggiunti- in diverse configurazioni- $\mathrm{i}$ bastoni di maresciallo, i sigilli di cancelliere, le chiavi di tesoriere e perfino le armate nel caso di generale d'artiglieria. Nel frattempo nei blasoni polacchi cominciaravano ad apparire anche prime e poche medaglie od insegne degli ordini cavalleresch $i^{13}$.

\section{Poche regole araldiche polacche}

Del resto nel modo rigoroso la nobiltà polacca rispettava l'uguaglianza proclamata per se all'interno del proprio stato. Lo dava l'espressione limitando il numero degli elementi nello stemma e per mancanza di preoccupazione circa il rispetto per il diritto occidentale araldico. Questo fenomeno si riflette anche nella costituzione del nostro parlamento del 1638 dove si ricorda nel modo chiaro che tutta la nobiltà sia uguale "de iure" e che pertanto qualsiasi titoli (ducali, di conte, di barone ecc.) siano proibiti ${ }^{14}$. Anche due costituzioni precedenti del 1613 e del 1633 ordinavano presentare certificati concreti insieme con la domanda di nobilitazione. Tale pratica mirava una prevenzione per non accettare gli uomini indegni nello stato di nobiltà ${ }^{15}$. Senza dubbio altra causa negativa nell'araldica polacca era piccolo affetto verso colori che erano utilizzati molto liberamente ${ }^{16}$. E' vero che in Polonia- a differenza d'Ovestle variazioni dei colori non formino gli stemmi separati e che un fondamento dell' identità di questi ultimi resti sempre l'emblema ${ }^{17}$. La povertà dei colori è stata unita con piccolo numero di parole usate per definire ogni colore nelle fonti polacche araldiche del Medioevo. Quindi il colore nero è stato chiamato di solito "niger", il verde- solo "viridis"ed inoltre è stato usato un erroneo termine "flaveus" per indicare così blu come giallo. Perció spesso non sappiamo quale colore era stato menzionato e rimane soltanto analisi delle note aggiuntive in latino per trarre una conclusione adeguata ${ }^{18}$. Altra nota caratteristica è la presenza del sopravvento chiaro del colore rosso nell'araldica polacca. Inoltre esiste una combinazione dei colori rosso-argento che puó giungere la nostra araldica con l'araldica svizzera ed austriaca ${ }^{19}$.

La mancanza dell'ufficio polacco d'araldo su modello inglese, francese o tedesco era un motivo importante per disattendere le regole araldiche nel nostro pa-

A. Kulikowski, op. cit., pp. 198-203.

„Volumina Legum”. Przedruk zbioru praw”, T. III, ed. J. Ohryzko, Petersburgo 1859, pp. 441-442: „O tytułach cudzoziemskich"

Ibidem, T. III, p. 83- "O nowej szlachcie” e p. 382- „O wywodzeniu szlachectwa”.

S. Orzechowski, „Żywot i śmierć Jana Tarnowskiego”, Sanok 1855, pp. 38-39.

J. Bieniak, op. cit., p. 197

S. Mikucki, „Barwa w heraldyce średniowiecznej”, parte I: „Herby rycerstwa zachodniego i polskiego”, [in:] "Rocznik Polskiego Towarzystwa Heraldycznego we Lwowie", T. 9 (1928/1929), pp. 221-222.

Ibidem, p. 225; P. Ganz, „Geschichte der heraldischen Kunst in der Schweiz im XII und XIII Jahrhundert”, Frauenfeld 1899, p. 39 
ese. Eppure tal ufficio vigilava una purezza dello stato cavalleresco per ufficiale registrazione e spiegazione delle armi araldiche, invece in Polonia non esisteva nessuna tale simile funzione. Per dire la verità dal 1403 deriva un certificato confermato l'esistenza del banditore a corte polacca, ma non ci sono conservate nessune traccie della sua attività. Anzidetto araldo si chiamava "Polanlant" cioè "Paese Polacco"-secondo il costume medievale per dare i pseudonimi dal nome del regno che c'era rappresentante per tale banditore ${ }^{20}$. Così divenne presto evidente che nel nostro paese sotto la protezione dei diritti siano soltanto le prerogative della nobiltànon i stemmi. Più tardi la mancanza di una protezione giuridica riguardava anche i cognomi. Pertanto se qualcuno sentiva offeso per ridicolizzare od insultare il suo blasone o cognome, doveva chiedere un risarcimento non in giudizio, ma individualmente- perfino con l'uso della forza contro il calluniatore ${ }^{21}$.

Tanto più si deve rilevare il fatto che dal paese, in cui non si attribuiva gran importanza al diritto araldico, provenivano numerosi stemmi cavallereschi e territoriali pubblicati nei medievali stemmari dell'Ovest ${ }^{22}$. Questo risulta anche dalla mancanza dei stemmari polacchi, perché come unico nostro stemmario medievale si tratta l'opera intitolata "Insygnia albo Klejnoty Królestwa Polskiego" creata negli anni 1464/1465-1480 ed attribuita a Giovanni Długosz. Tutti altri stemmari sono già creati in Polonia dell'epoca moderna-tra cui i più famosi di Bartosz Paprocki e di Gaspare Niesiecki ${ }^{23}$.

Del resto i blasoni erano situati anche sulle varie opere dell'arte (come in Occidente), ma particolarmente preziosi- soprattutto per i genealogisti-restano ad oggi quelli che sono visibili sulle lapidi, epitaffi e tavole di fondazione, perché soddisfano il ruolo dei simboli che identificano le persone concrete ${ }^{24}$. Dalla fine del XV secolo si cominciò a localizzare i stemmi sulle lapidi secondo un ordine rigoroso- cioè, in sostanza, quattro stemmi- uno in ogni angolo. Tuttavia non c'era molteplice im-

A. Nadolski, „Broń i strój rycerstwa polskiego w Średniowieczu”, Breslavia-Varsavia-Cracovia-Danzica 1979, pp. 118-119; W. Łoziński, „Życie polskie w dawnych wiekach”, Cracovia 1964, p. 156; S.K. Kuczyński, „Herby w twórczości historycznej Jana Długosza”, [in:] „Sztuka i ideologia XV w.”, ed. P. Skubiszewski, Varsavia 1978, p. 224. II documento del banditore polacco di 25.VIII.1403 é stato pubblicato in: „Zbiór dokumentów małopolskich", parte VI, ed. I. Sułkowska-Kuraś e S. Kuraś, Breslavia-Varsavia-Cracovia-Danzica 1974, documento $\mathrm{nr} 1658$.

21 P. Dąbkowski, „Prawo prywatne polskie”, T. I, Leopoli 1910, p. 97; J. Matuszewski, „Polskie nazwisko szlacheckie", Łodz 1975, pp. 109-111.

22 Vedi: breve descrizione di questi stemmari in: A. Heymowski, „Polish arms in medieval armorials”, [in:] „The Coat of Arms", T. VII (Londra 1964], nr 56, pp. 57-62; lo stesso autore, "Les cimiers médiévaux...", op. cit., pp. 132 e seg.; lo stesso autore, „Rycerstwo polskie w zachodnich herbarzach XIV i XV stulecia”, [in:] „Prace Kongresu Kultury Polskiej”, T. VIII („Polskie więzi kulturowe na Obczyźnie”), ed. M. Paszkiewicz, Londra 1986, pp. 2836; J. Szymański, op. cit., pp. 12-17, J. Łojko, op. cit., pp. 24-27 e S.K. Kuczyński, „Polskie herby ziemskie...”, op. cit., pp. 47-57.

23 Vedi: M. Kazańczuk, „Staropolskie herbarze. Herby- historia- religia”, [in:] „Pamiętnik Literacki”, R. XCIII (2002), quaderno nr 3, pp. 37-57. La si trova anche vasta bibliografia adeguata.

24 J. Łojko, op. cit., pp. 90-96; P. Mrozowski, „Polskie tablice erekcyjne z wieków XIV i XV”, [in:] „Studia Żródłoznawcze”, T. XXII/XXIII (1990), pp. 77-112; lo stesso autore, „Polskie nagrobki gotyckie”, Varsavia 1994, passim; M. Walicki, „Malarstwo polskie: Gotyk, Renesans, wczesny manieryzm”, Varsavia 1963, pp. 293-294. 
magine araldica della stessa persona, ma quattro immagini degli stemmi di quattro persone differenti: del padre del defunto (cioè proprio stemma gentilizio), della madre, della nonna da parte di padre e della nonna da parte di madre che-conforme alla legge ed al costume- permetteva già documentare la propria nobiltà ${ }^{25}$. Seconda tipica composizione prevedeva scolpire cinque stemmi araldici nella pietra tombale (quattro agli angoli e quello centrale ), in cui lo stemma in posizione centrale era di padre (cioè proprio stemma gentilizio) e fra altri si trovava anche questo di bisnonna da parte di padre ${ }^{26}$. Del resto tutte e due composizioni erano molto simili alla presentazione delle unioni familiari nel caso degli stemmi con quattro o cinque campi (cioè degli stemmi composti) (vedi: illustr. nr 4). Naturalmente una lapide potrebbe mostrare solo unico blasone o perfino molti insieme, peró c'erano configurazioni già meno frequenti. Anche ci sono state le combinazioni di stemmi del marito $\mathrm{e}$ della moglie e dei loro antenati. Infine i blasoni erano scolpiti sulle lapidi dei bambini. Lo suggeriva che il bambino sia stato trattato come un membro "a pieno titolo" di famiglia ed anche come un membro della comunità di stirpe. Fra i ricercatori ci sono pure tali concetti che la presenza degli stemmi era solo un problema artistico per riempire lo spazio vuoto nella composizione d'una lapide ${ }^{27}$.

Quindi è solo un passo per fissare total età maggiore della persona nel Medioevo. A quel tempo tale persona rompeva definitivamente con l'infanzia e riceveva automaticamente il diritto d'eseguire tutte azioni legali. Nella vecchia Polonia quest' età era fissata a 24 anni e non dipendeva da nessuna modificazione. Invece sempre si cambiava "l' età efficiente"cioè il periodo di transizione tra infanzia e piena età adulta. Durante questo periodo l'uomo giovane poteva eseguire la maggioranza delle azioni legali (perfino sposarsi), ma con l'eccezione di cessione perenne dei beni immobili. Inizialmente quest' "età efficiente"non è stata determinata mai e come unico criterio si usava la maturità fisica. Tuttavia nel tardo Medioevo un limite di quest'età è stato fissato per 12 anni e poi tal limite aumentava sistematicamente fino al 1768 , quando raggiungeva 20 anni definitivamente ed indipendemente dal $\operatorname{sesso}^{28}$.

\section{Il processo della creazione di cognomi polacchi}

Un altro interessante fenomeno genealogico sembra essere un processo della creazione di nomi polacchi, sebbene esso abbia gli elementi comuni con l'araldica

J. Łojko, op. cit., pp. 96-98; W. Semkowicz, „Nagana i oczyszczenie szlachectwa w Polsce w XIV i XV w.”, Leopoli 1899, pp. 20-39; S. Konarski, „O polskich herbach złożonych”, [in:] „Miesięcznik Heraldyczny”, R. XI (1932), pp. $35-37$.

W. Dworzaczek, op. cit., p. 95. Tutte e due composizioni dei 4 e 5 blasoni si incontreremo anche nei campi delle volte, nelle mensole e nei tamburi delle cupole nel caso delle cappelle tombali e dei mausolei familiari- vedi: J. Łoziński, „Grobowe kaplice kopułowe w Polsce (1520-1620)”, Varsavia 1973, pp. 63-70. M. Kołakowska, „Renesansowe nagrobki dziecięce w Polsce XVI w. i I połowy XVII w.”, [in:] „Studia Renesansowe”, T. I (1958), pp. 245-254. 
per l'uso i proclami degli stemmi (in latino: "proclamatio"). Essi sono uno degli elementi essenziali dello stemma polacco e spesso sono identificati semplicemente con il suo nome, ma non occorre confonderli con il motto che-come "una sapienza della vita"- si pubblica di solito sul nastro sotto lo scudo. Per dire la verità i proclami erano gli slogan, gridi di battaglia, invece nel blasone non esistevano visualmente mai. Essi funzionavano esclusivamente come "i segni acustici" dello stemma. Anche il nome del blasone non doveva sempre essere d'accordo con questo grido, perchè spesso lo stemma possedeva il proprio nome-per esempio: anche oggi stemma "Ślepowron" ha proclama "Bujny" e stemma "Topór"-proclama "Starża". Da alcuni gridi procedono i nomi nobiliari, ma senz'altro non tutti. Appunto i proclami personali creano il più grande gruppo che si divide ai gridi: a) nominali (dal nome d'un membro eminente della stirpe o dal nome del suo capostipite-per esempio: "Bończa" da "Boniek"); b) soprannominati (dal soprannome o dal predicato d'uno degli antenati di tutta stirpe-per esempio: "Pierzchała", "Grzymała", "Momot" ecc.; c) etnici (dalla provenienza della stirpe-per esempio: "Sas", "Prus" ecc.). Numerosi sono anche i proclami topografici che derivano dai nomi specifici di luoghi (dove si trovava un nido gentilizio), di terre e perfino di fiumi e di laghi circostanti-per esempio: "Nałęcz", "Szreniawa", "Odrowąż", "Bogoria". Infine il più piccolo, terzo gruppo creano i proclami che funzionavano come i gridi di combattimento-per esempio: "Zerwikaptur", "Ostoja", "Bożawola", "Doliwa", "Dołęga" ecc. ${ }^{29}$

Mentre in Italia le prime tracce di cognomi si puó trovare già nel circa 800 , in Polonia l'uso generale di cognomi si comincia solo dal XIX secolo. Una testimonianza di questo fenomeno puó essere la decisione del 27 marzo 1821 pubblicata nel Regno di Polonia e con l'ordine per nel corso di sei mesi dare i cognomi per tutti questi uomini che non possedono ancora nessuno cognome ${ }^{30}$. Specie lento era un processo di preso dei cognomi fra la popolazione contadina ed ebraica ${ }^{31}$.

Dobbiamo anche ricordare che per conoscere il processo della formazione di cognomi sia sempre necessario rivedere il gran numero di fonti storiche. Solo in tal modo possiamo trovare i fenomeni concreti che creavano i cognomi. Quindi di quali fenomeni possiamo dire in Polonia?

1) L'eredità del cognome di padre per i bambini (eventualmente lo stesso cognome preso dal nonno) cioè l'ereditarietà dei cognomi almeno per tre generazioni. Anche si prende in considerazione un cognome comune di padre, di

29

Dei proclami scrivono vastamente: J. Szymański, op. cit., pp. 61-65; A. Kulikowski, op. cit., pp. 203-207; J. Łojko, op. cit., p. 17.

„Dziennik Praw Królestwa Polskiego”, T. VII (1821), nr 30, pp. 134 e seg. Anche vedi: J. Matuszewski, op. cit., p. 27; S. Grzybowski, „Nazwisko i jego stałość jako elementy identyfikacji osoby w dawnym prawie polskim”, [in:] „Onomastyka”, R. III (1957), quaderno nr 2, pp. 490-491 e 509.

S. Grzybowski, op. cit., p. 491. 
madre e di figlio ed inoltre il caso quando esiste tale cognome fra i coniugi al plurale-ad esempio: i signori Zamoyski, i signori Lis ecc.

2) L'identificazione tutta la generazione per un cognome comune. Si tratta dei fratelli ed eventualmente delle sorelle (se sono non sposate).

3) L'accettazione il cognome del marito dalla moglie.

4) L'identificazione dei parenti lontani per un cognome comune.

Questi fenomeni erano relativi ai membri di tutti stati ad eccezione del clero dove eppure non si conosce le regole di successione all'interno della famiglia e dove sacerdozio (o voto religioso) sostituisce l'istituzione del matrimonio ${ }^{32}$. Inoltre è caratteristico che mentre in Italia, in Francia od in Germania (e dove il patriziato urbano aveva un ruolo importante) gli aristocrati conservavano la loro particella nobiliare "di", "de" o "von", in Polonia non si usava nessun modo linguistico per accentuare la propria nobiltà. La particella "-ski"- che in origine potrebbe avere tali funzioni- in realtà è stata democratizzata completamente, perché essa era portata per tutta la folla di persone non necessariamente derivate dalla nobiltà ${ }^{33}$.

\section{I segni borghesi in Polonia}

Alla fine vale la pena di descrivere appunto i segni borghesi che nelle condizioni polacche erano conosciuti prevalentemente come "gmerk", ma anche come ,herbik”, „signum”, ,signetum”, ,Zeichen”, „,character” (dal latino o dal tedesco). Ciò aveva la sua spiegazione, perché la maggioranza delle città polacche era abitata per il patriziato tedesco- soprattutto in Slesia ed in Pomerania ${ }^{34}$. Tuttavia al tempo stesso non si puó trattare questi segni come gli stemmi gentilizi- anche se spesso erano simili per l'uso dei motivi simili d'origine vegetale, animale od umana. Del resto in generale dopo 1300 erano creati ancora altri elementi dello stemma come l'elmo e la gemma (e poi anche: lambrecchini, corone, manti ecc.), invece i segni borghesi hanno sostato nella propria evoluzione. I marchi nella forma dei righi (collegati con le rune scandinave e slave) ed anche i marchi presentati gli attrezzi del lavoro o le merci del proprietario (che di regola suggerisce un'appartenenza della persona adeguata ai mercanti od agli artigiani) creavano peró uno gruppo significativo di questi "gmerk" 35 . p. 312

Z. Kowalik-Kaleta, „Historia nazwisk polskich na tle społecznym i obyczajowym (XII-XV w.)”, T. I, Varsavia 2007. J. Matuszewski, op. cit., p. 136.

Vedi il vasto studio sul tema di segni borghesi nelle città polacche: A. Małecki, „Studia heraldyczne”, T. II, Leopoli 1890, pp. 324-385 e 387-393 (nelle pagine ultime ci sono perfino 4 tavole con le immagini di questi segni). Ibidem, p. 347. Inoltre: M. Gumowski, „Herbarz patrycjatu toruńskiego”, Torunia-Posnania 1970, pp. 6 e seg.; W. Maisel, „Archeologia prawna Polski”, Posnania 1982, p. 277. 
Era anche conosciuto una compenetrazione fra stemmi gentilizi e segni borghesi in vari territori- perfino molto allontanati da sè. A. Małecki presenta appunto tal esempio dopo l'analisi del contenuto d'una vecchia stampa dal 1657 e suppone che là presentate ${ }^{35}$ immagini araldici si siano impossessati per i cittadini di Norimberga dalla nobiltà polacca o viceversa: che i polacchi li rubi da questo patriziato tedesco. Differenze nei dettagli iconografici sono stati infatti davvero minimali ${ }^{36}$.

Un altro esempio interessante è l'uso del segno borghese nello stemma "Geometer" che è stato creato nel 1582 come risultato di nobilitazione del geometra reale (e simultaneamente dell'ufficiale servente in esercito polacco) Pietro Franco ("Francus" in diploma). Il documento di nobilitazione non onora i genitori di Franco per la parola "nobiles" o per l'altra tipica per la nobiltà, ma in cambio di questo trasferisce precedenti "insegne" familiari (qui in forma della gamba alata di grifone) nella gemma, sopra la corona araldica nel nuovo blasone. Tale pratica suggerisce chiaramente un'origine borghese della famiglia italiana di Franco. Inoltre a quest'occasione abbiamo anche da fare con l'adozione d'arme, perché come emblema è stato dato una varietà dello stemma "Jelita" e ciò ha avuto luogo su richiesta adeguata del comandante dell'esercito polacco Giovanni Zamoyski (vedi: illustr. nr 5) ${ }^{37}$.

\section{La conclusione}

Come vediamo, il diritto polacco genealogico ed araldico è pieno di sfumature diverse che lo distinguono dalle regole obbligate in Occidente. Attualmente cresce l'interesse di questi campi della storia ed anzidetto diritto rivive in epoca d' informatizzazione. Anche belli stemmi colorati e tavole genealogiche acquisiscono una nuova luce sugli schermi dei computer quando sono fatte nuove pagine web per una folla dei lettori fedeli. La specificità del diritto polacco genealogico ed araldico consisteva invece nel numero limito degli elementi nello stemma e nella mancanza di preoccupazione circa il rispetto per il diritto occidentale araldico. In alcuni stemmi polacchi si può vedere perfino i segni conosciuti dall'epoca di primi stirpi feudali. Anche originale è il processo della creazione di cognomi polacchi- conosciuto poco in Europa Occidentale.

37 A. Małecki, op. cit., T. II, p. 357; B. Paprocki, „Herby rycerstwa polskiego”, Cracovia 1858, pp. 282-283; J. Szymański, „Herbarz rycerstwa polskiego z XVI w.”, Varsavia 2001, pp. 70-71. 


\section{SPECYFIKA POLSKIEGO PRAWA GENEALOGICZNEGO I HERALDYCZNEGO}

Praktycznie każdy kraj europejski posiada swoją tożsamość kulturową ukształtowaną przez historię, a to właśnie wyciska piętno na znakach i symbolach, co łączy się też z poczuciem narodowej czy rodzinnej dumy. Naprzeciw temu powszechnemu zapotrzebowaniu wychodzą nauki pomocnicze historii, a w szczególności genealogia i heraldyka. Obie te dziedziny wiedzy są bowiem nie tylko bardzo ,widowiskowe" ze względu na możliwość tworzenia drzew genealogicznych oraz herbów, ale również wpisują się znakomicie w ogólnoświatowy ruch genealogiczno-heraldyczny, jaki pojawił się na przełomie lat 80 -tych i 90-tych XX w. w związku z wynalezieniem komputera osobistego i Internetu. Ta rewolucja informatyczna doprowadziła do tego, że powstały całkiem nowe sposoby gromadzenia, przechowywania i prezentowania danych na temat pochodzenia rodów, rodzin czy ich herbów. Równolegle do tego zjawiska nastapiła "fala" nowych, kolorowych i popularnych wydawnictw genealogicznych i heraldycznych, które ciągle znajdują licznych nabywców w gronie osób zainteresowanych badaniem swoich „korzeni”.

Należy jednak pamiętać, iż wspomniane nauki pomocnicze historii mają wiele wspólnego z prawem, bo nie da się odtwarzać herbu czy wywodu swoich przodków bez znajomości reguł ustalonych kilka wieków temu i odnoszących się do tych konkretnych czynności. Co więcej: w Polsce wczesnośredniowiecznej pojawiły się pewne ważne procesy społeczne, które w ówczesnej Europie Zachodniej przebiegały inaczej i znacznie szybciej, a w konsekwencji takie zjawisko musiało doprowadzić do powstania specyficznych cech polskiej genealogii i heraldyki. Mowa tutaj o znacznie silniejszym niż na Zachodzie poczuciu wspólnoty plemiennej i rodowej, co powodowało, iż powstające rycerstwo polskie czuło się silniej związane ze swoim rodem - posiada jącym jednego, wspólnego przodka - niż z własną ziemią. W ten sposób dochodziło do takich sytuacji, że podczas gdy np. we Włoszech tylko jedna rodzina - powiązana faktycznymi i udokumentowanymi więzami krwi - posługiwała się herbem Colonnów czy Orsinich, to w Polsce mamy np. herby „Jelita” czy „Nałęcz”, którymi pieczętowało się kilkaset rodzin, w ogóle ze sobą nie spokrewnionych, ale powołujących się na wspólnego przodka - choćby i legendarnego.

Z przynależnością do jednego rodu wiązało się ściśle zawołanie (czyli proklama - ,proclamatio"), które jest cechą charakterystyczną jedynie dla heraldyki polskiej. O ile bowiem na Zachodzie wystarczał jedynie sam wizerunek herbu, o tyle u nas stosowano jeszcze wersję słuchową takiego wyobrażenia. Zawołanie stano- 
wiło nazwę rodową i było używane zawsze równolegle do herbu, choć nie zawsze brzmienie zawołania da się utożsamić z nazwą samego herbu.

Na polskich średniowiecznych herbach zamieszczano też symbole pochodzenia starosłowiańskiego ryte na pniach drzew, naczyniach ceramicznych, urnach czy broni. Symbole te miały charakter run, a nawet znaków magicznych widniejących na totemach. Część z nich do dzisiaj nie może doczekać się rozszyfrowania i nosi specyficzne nazwy.

Odrębnym zagadnieniem są wreszcie mieszczańskie gmerki- porównywane niekiedy do herbów szlacheckich, ale występujące głównie w polskich miastach opanowanych przez niemiecki patrycjat (Gdańsk, Toruń, Elbląg, Poznań itp.), więc najprawdopodobniej nie stanowiące wytworu naszej rodzimej heraldyki.

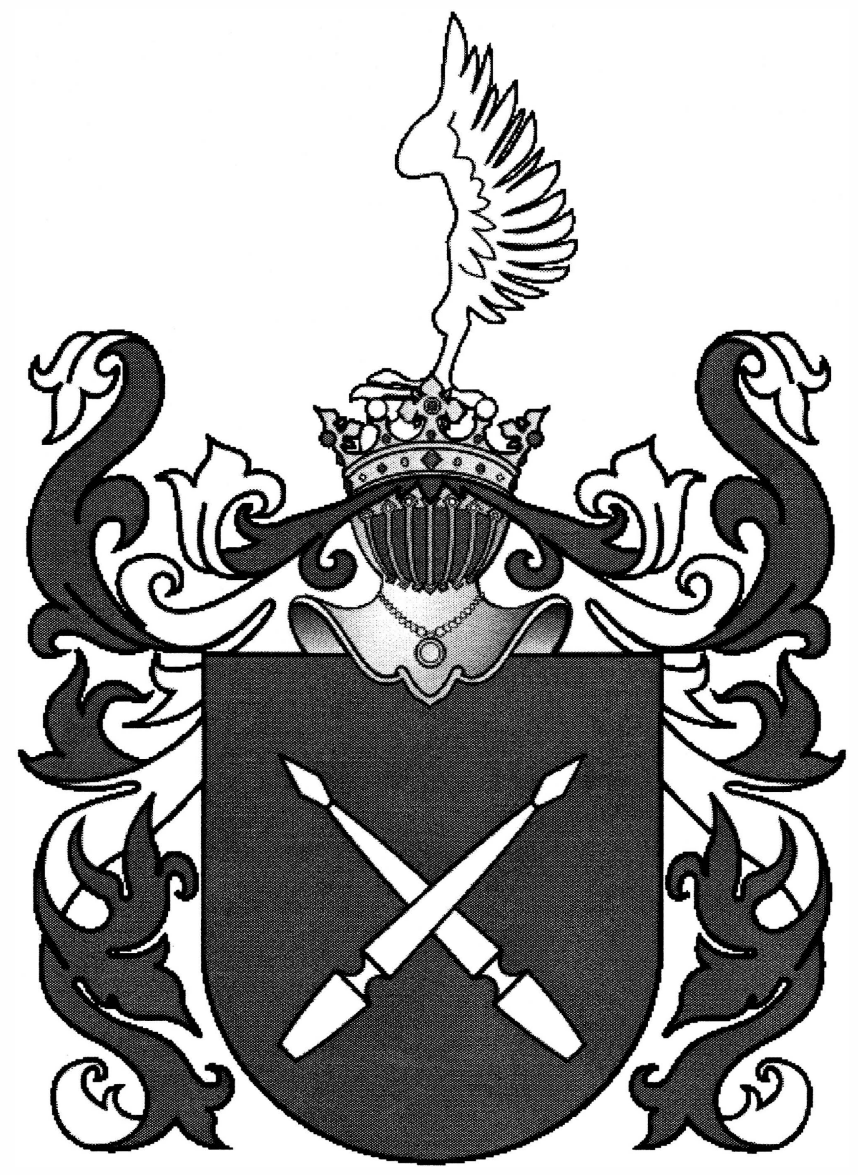



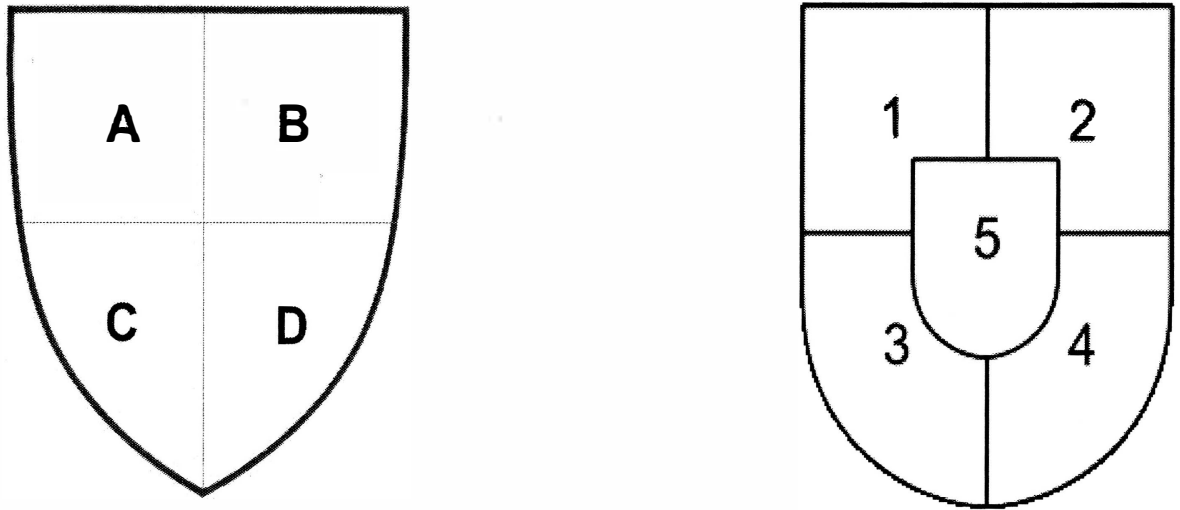

Dislocazione degli stemmi polacchi sulle lapidi e sugli stemmi composti del XIV-XVI secolo: Tipo I: A- stemma di padre, B- di madre, C- di nonna da parte di padre, D- di nonna da parte di madre.Tipo II: 1- di madre, 2- di nonna da parte di padre, 3- di nonna da parte di madre, 4- di bisnonna da parte di padre, 5- di padre.

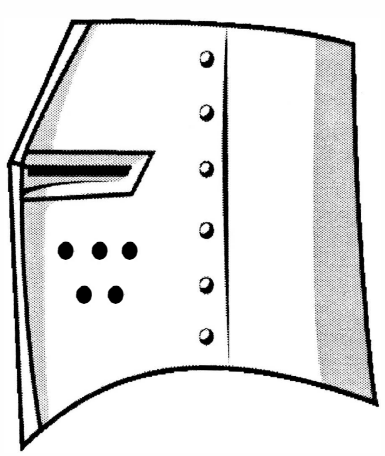

elmo pentolare

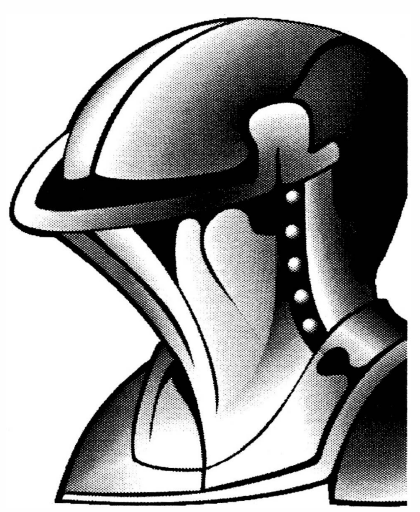

„bocca di rana”

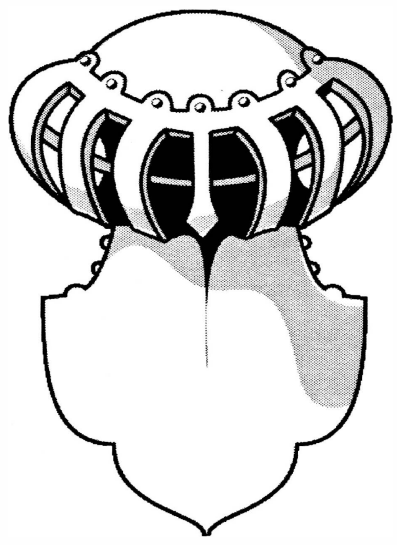

elmo di barra 


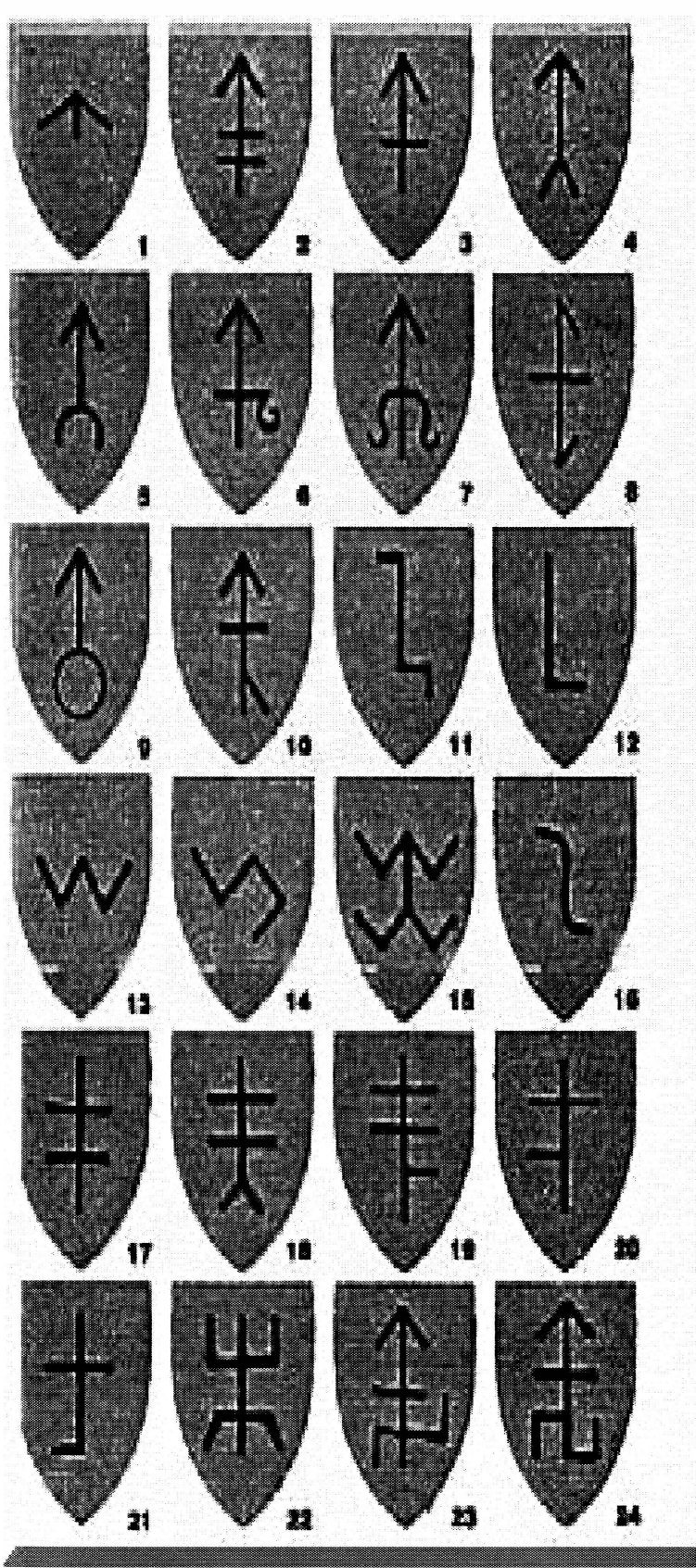

1. Źieleźce strzaty (Pogoria)

2. Strzata dwakroć przekrzyżowana (Lis)

3. Strzala przekrzyżowana (Kościeszavel Strzegonia Chodkiewiczów)

4. Strzała dolem rozdarta (Sas)

5. Strzała na barku pierścienia (Ogończyk, Ostrogscy-Zastawscy)

6. Krzyzostrzala a strzała przekrzyżowana $z$ wasem

7. Odrowaz a odrzywas (Odroważowi)

8. Pót strzaty (a hak) przekrzyżowane

9. Strzała na pierścieniu (ogniwie)

10. Strzała przekrzyżowana z podpora

11. Garda a jelec

12. Winkiel (Norwid)

13. Dwie krokwie zlaczone (Adbank)

14. Dwie krokwie, jedna zlamana

15. Dwie krokwie stupkiem złaczone

16. Krzywaśn velkij krzywy (Sieniawa Lubomirskich)

17. Krzyż podwójny (na tarczy jeźdźca Pogoni Litewskiej)

18. Krzyż podwójny dolem rozdarty

19. Póttrzecia krzyża (Pilawa Potockich)

20. Póltora krzyża (Prus)

21. Krzyż złamany

22. Widty

23, 24. Typy znaków (runów?) bez na$z w y$, określanych $w$ herbarzach stowami. ,znak widoczny na rysunku" 


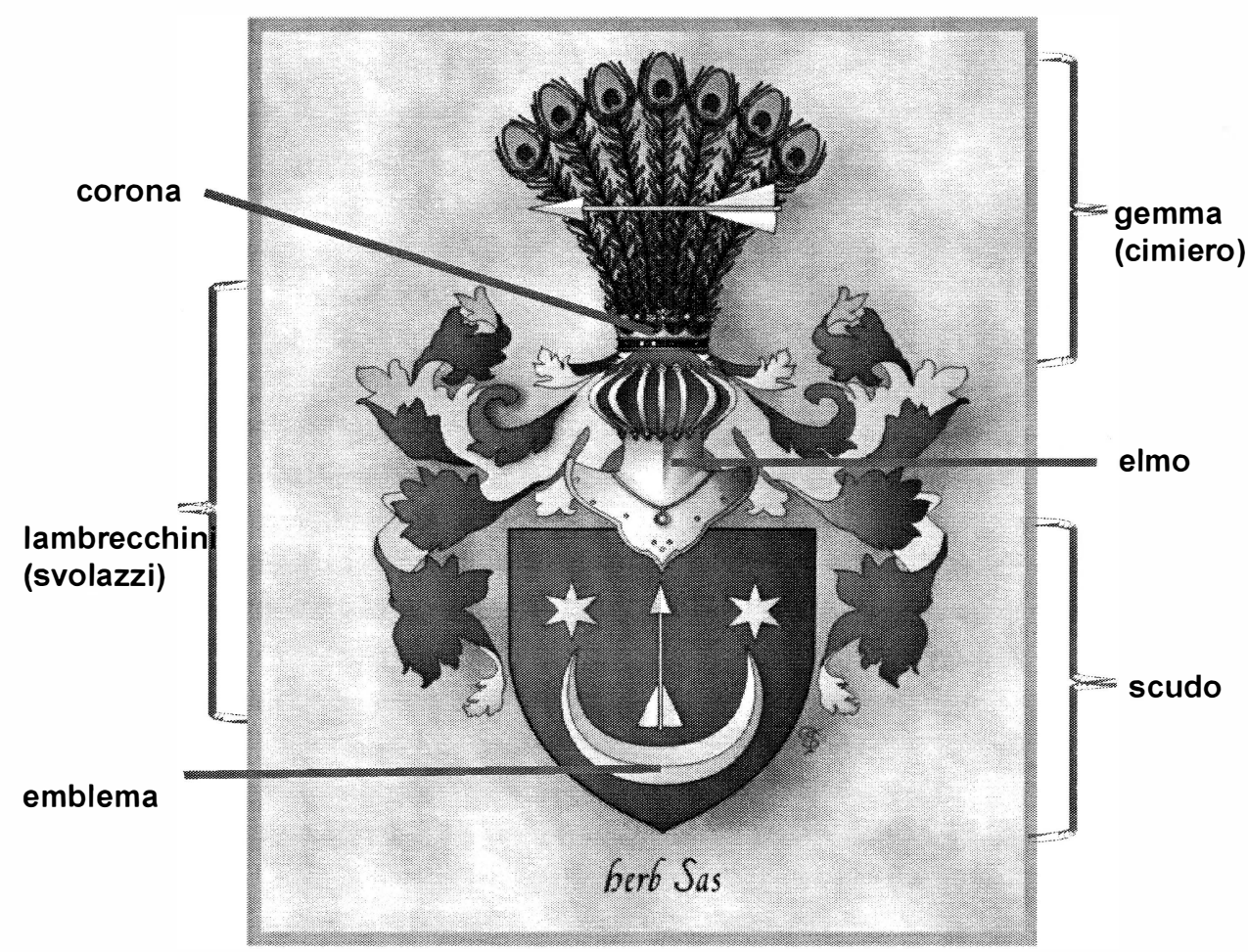




\section{SPECIFICITY OF POLISH GENEALOGICAL AND HERALDIC LAW}

Every European country has its cultural identity shaped by history, and it impresses the signs and symbols, and connects with the sense of national and family pride. Sciences supporting history, and in particular its genealogy and heraldry, meet that universal demands.

Both of these fields of knowledge are not only very "spectacular" because of the possibility of creating trees and coats of arms, but also enter perfectly into a worldwide genealogical movement, which appeared in the late 1980s and 1990s of the 20th century, largely due to the invention of the personal computer and the Internet. This revolution in computer science led to quite new ways in the collection, storage and presentation of data on family origins and their coats of arms. In parallel with this phenomenon, a "wave" of new, colorful and popular publications concerning genealogical and heraldic issues appeared, which today, still hold the attention of people interested in testing their "roots".

Key words: genealogy, heraldry, coat of arm, revolution in computer science, Internet 